\title{
Material Basis and Mechanism of Chansu Injection for COVID-19 Treatment Based on Network Pharmacology and Molecular Docking Technology
}

\author{
Yong Xu $\mathbb{B}^{1},{ }^{1}$ Wenpan Peng ${ }^{\mathbb{D}},{ }^{1}$ Di Han, ${ }^{1}$ Zhichao Wang, ${ }^{1}$ Fanchao Feng, ${ }^{1,2}$ \\ Xianmei Zhou $\mathbb{D},{ }^{1,2}$ and Qi Wu $\mathbb{D}^{3}$ \\ ${ }^{1}$ Affiliated Hospital of Nanjing University of Chinese Medicine, Nanjing 210029, China \\ ${ }^{2}$ Department of Respiratory and Critical Medicine, Jiangsu Province Hospital of Chinese Medicine, Nanjing 210029, China \\ ${ }^{3}$ Department of Physiology, Xuzhou Medical University, Xuzhou 221009, China
}

Correspondence should be addressed to Xianmei Zhou; zhouxianmeijs@aliyun.com and Qi Wu; wuqi@xzhmu.edu.cn

Received 6 July 2021; Revised 30 August 2021; Accepted 8 September 2021; Published 11 October 2021

Academic Editor: Talha Bin Emran

Copyright $\odot 2021$ Yong Xu et al. This is an open access article distributed under the Creative Commons Attribution License, which
permits unrestricted use, distribution, and reproduction in any medium, provided the original work is properly cited.

Purpose. The clinical efficacy of Chansu injection for COVID-19 treatment has been confirmed. Its mechanism of action remains unclear. We used network pharmacology and molecular docking technology to explore the potential material basis and mechanism of action of Chansu injection for COVID-19. Methods. The main components of Chansu injection were determined using HPLC. The PharmMapper, SwissTargetPrediction, SEA, and TCMID databases were used to screen for the active ingredients and therapeutic targets of Chansu injection, while the OMIM and GeneCards Suite databases were used to search for COVID-19-related targets. The STRING database was used for protein-protein interaction (PPI) network construction and topological analysis, while DAVID was used for Gene Ontology (GO) and Kyoto Encyclopedia of Genes and Genomes (KEGG) pathway enrichment analyses of the core targets. The main active compounds of Chansu injection were docked with 3CL protease, ACE2, RdRp, and spike protein. Results. The three Chansu injection compounds were identified using HPLC. A total of $236 \mathrm{drug}-$ related targets and 16,611 disease-related targets were identified, and 77 common targets were determined through mapping. The PPI mapping results revealed that 16 core targets were obtained through topological analysis and screening. Furthermore, GO and KEGG pathway enrichment analyses revealed that the PI3K and JAK-STAT signaling pathways are the major pathways. The molecular docking results suggest that the three Chansu injection components have high binding energies to the $S$ protein. Conclusions. The potential mechanism of Chansu injection for COVID-19 involves multiple targets and pathways, thereby providing a scientific basis for its clinical application and further research.

\section{Introduction}

Coronavirus disease 2019 (COVID-19) caused by severe acute respiratory syndrome coronavirus 2 (SARS-CoV-2) emerged in China in November 2019 [1]. This disease has become a global pandemic with severe health issues around the world $[2,3]$. This is the third pandemic that has occurred during the last 20 years of the 21 st century, the first of which was the SARS pandemic in 2003, and the second, Middle East respiratory syndrome (MERS) pandemic in 2015 [4-6]. Worldometers real-time statistics show that as of February 1, 2021, SARS-CoV-2 has caused more than 100 million infections and more than 2.22 million deaths. As a result, it is considered a public health emergency of international concern [7-9].

SARS-CoV-2 is the seventh coronavirus found to infect humans. Similar to SARS-CoV and MERS-CoV, SARS$\mathrm{CoV}-2$ belongs to the $\beta$-subtype coronavirus [10]. Its genetic similarity to that of the bat coronavirus is $96 \%$, and it shares the same cell receptor, angiotensin-converting enzyme II (ACE2), found in SARS-CoV. SARS-CoV-2 is a singlestranded RNA with the largest RNA virus $[11,12]$. It can trigger an antiviral immune response upon entry into organisms, causing slight or moderate respiratory symptoms 
that are difficult to notice in most patients. Severe cases display excessive inflammatory responses, leading to severe progressive pneumonia and even acute respiratory distress syndrome, septic shock, coagulation disorders, and multiorgan failure $[13,14]$. Owing to its severity and rapid progression, there is an urgent need to develop novel medicines for the emerging coronavirus [15].

Although inactivated viral vaccines, adenoviral vector vaccines, recombinant protein vaccines, and mRNA vaccines have been intensively developed in various countries, they are also in use and have achieved good efficiency indicators [16]. However, vaccination and development are also difficult [17]. The problem with common viral vector vaccines is that many people have already developed an immune response to these viral vectors, and these neutralizing antibodies against adenovirus are likely to have an impact on the effectiveness of the vaccine. Inactivated viral vaccines not only require adjuvants but also often require multiple doses to boost immunity. The efficacy of the ground-breaking mRNA vaccine in humans needs to be further tested, and whether it can be delivered through the mucous membranes of the respiratory tract is also a question worth exploring. In the face of long vaccine development and vaccination cycles, and with little success in acute COVID-19 patients, there is an urgent need for an effective antiviral drug that can mitigate disease progression in acute patients or in vaccine-free areas.

However, to date, no medicines specific for coronavirus treatment are available. Before the COVID-19 pandemic in China, the coverage and effectiveness of Chinese medicines were greater than $90 \%$ [18]. Results of clinical observation revealed that Chinese medicine could effectively mitigate disease progression, stop the progression from mild to severe, increase the recovery rate, decrease the death rate, and promote rehabilitation [19]. The full participation of Chinese medicine has become a highlight of the anti-COVID-19 campaign in China and has contributed to the pandemic's global campaign. As a result, Chinese medicines have been increasingly acknowledged.

Chansu is obtained from the excreta of the skin and postauricular glands of Bufo bufo gargarizans. Chansu injection is a prescription Chinese medicine, its main component is a derivative of indole base, and this product is a colorless to light yellow clear liquid, often used for clearing heat and detoxifying effect. It fights inflammation and infection and regulates immunity $[20,21]$. Chansu injection is now being widely administered to inhibit bacteria, fight viruses, suppress pain, reduce inflammation, inhibit the proliferation of tumor cells, increase the patient's albumin level after radiochemical therapy, and induce tumor cell apoptosis. Favorable clinical therapeutic efficiency and safety assessments have also been acquired in the ancillary treatment of cancer and chronic hepatitis. The main active ingredients in Chansu, such as bufalin, resibufogenin, and cinobufagin, have very strong antiviral effects $[22,23]$. In a clinical trial, Fen et al. found that Chansu injection significantly improved the respiratory function of patients with severe COVID-19, suggesting a marked clinical effect and no side effects [24].
Network pharmacology is an emerging discipline that integrates the technology and content of polypharmacology, computational biology, and network analysis [25]. Because the integrality and systematicity of network pharmacology are consistent with the Chinese medicine theory [26], it has been widely used in the prediction and characterization of potential targets and active ingredients of Chinese medicine [27]. Molecular docking technology is a theoretical simulation approach to predict the binding pattern and affinity of medicines using computers to study the receptors' characteristics as well as the interaction between receptors and medicinal molecules [28]. In this study, we attempted to identify the effective ingredients and targets of Chansu injection for COVID-19 treatment using a network pharmacological method. Molecular docking was also applied in investigating the binding capacity of protein receptors to small medicine molecules based on geometric mapping and energy mapping, a key-and-lock principle. The material basis and molecular mechanism of Chansu injection for COVID-19 treatment were explored. A flowchart of the research methodology is presented in Figure 1.

\section{Materials and Methods}

2.1. Database. PharmMapper (https://lilab-ecust.cn/phar mmapper/), Universal Protein (UniProt, https://www. uniprot.org/) database, similarity ensemble approach (SEA, https://sea.bkslab.org) database, Swiss database (SwissTargetPrediction, https://www.sib.swiss), STRING (https://string-db.org), the Database for Annotation, Visualization, and Integrated Discovery (DAVID, https:// david.ncifcrf.gov), GeneCards database platform (https:// www.genecards.org), Comparative Toxicogenomics Database (CTD, https://ctd.mdibl.org), and Therapeutic Target Database (TTD, https://bidd.nus.edu.sg/group/ttd/ttd.asp) were used.

2.2. Ingredient Analysis. The Chansu injection sample was supplied by the Pujin Pharmaceutical Cooperation (Jiangsu, China). The chromatographic conditions and system compatibility tests included the Agilent Zorbax SB-C18 chromatographic column (column length $250 \mathrm{~mm}$, inner diameter $4.6 \mathrm{~mm}$, and particle size $5 \mu \mathrm{m}$ ) and the mobile phase containing acetonitrile and $0.1 \%$ ammonium acetate solution (50:50). The detection wavelength was $296 \mathrm{~nm}$. Theoretical plate counts were calculated according to the bufalin level peak, which was not less than 3,000. To prepare the control solution, an appropriate amount of bufalin, resibufogenin, and cinobufagin was calibrated precisely and $1.6 \mu \mathrm{g}$ each of bufalin, resibufogenin, and cinobufagin were added to $1 \mathrm{~mL}$ of $50 \%$ acetonitrile aqueous solutions. The control solution $(20-50 \mu \mathrm{L})$ and $50 \mu \mathrm{L}$ of the test samples were loaded into high-performance liquid chromatography (HPLC) equipment, and the calculations were derived with an external method. Each $1 \mathrm{~mL}$ of sample should contain 1.2-4.8 $\mu$ g of bufalin $\left(\mathrm{C}_{24} \mathrm{H}_{34} \mathrm{O}_{4}\right)$, cinobufagin $\left(\mathrm{C}_{26} \mathrm{H}_{34} \mathrm{O}_{6}\right)$, and resibufogenin $\left(\mathrm{C}_{24} \mathrm{H}_{32} \mathrm{O}_{4}\right)$. 


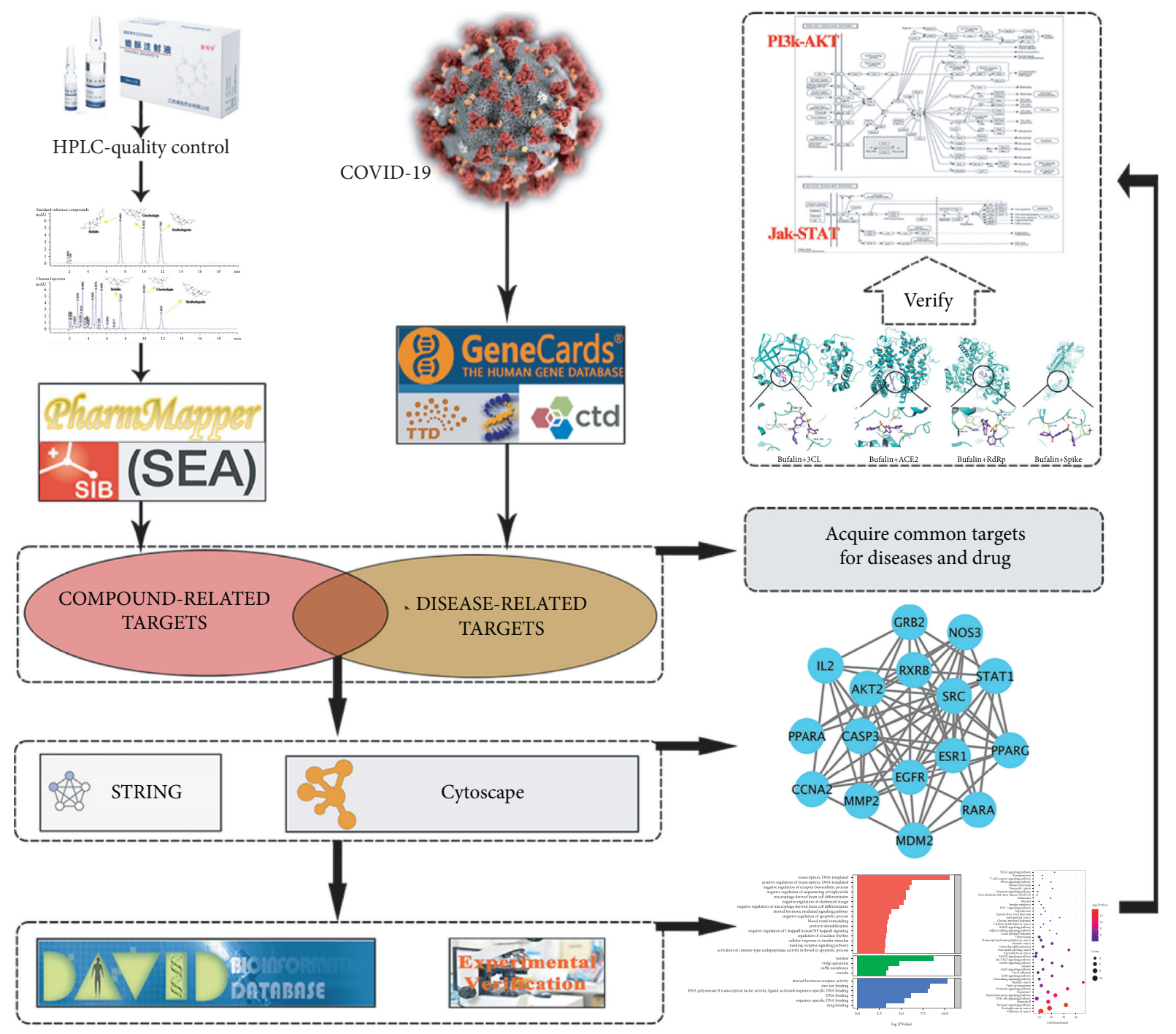

Figure 1: Flowchart of the study.

2.3. Acquisition of Potential Targets. Potential targets of the active ingredients of Chansu injection were searched online on PharmMapper, SEA, and SwissTargetPrediction, using the index words bufalin, resibufogenin, and cinobufagin. The combined results were considered potential targets.

2.4. Acquisition of Targets for COVID-19. The index words coronavirus disease, coronavirus infections, and severe acute respiratory syndrome were searched in CTD, TTD, and GeneCards Suite, respectively. The index word search was conducted on January 20, 2021. The combined results of the three databases were used, and duplications were removed to obtain the corresponding targets for COVID-19.

2.5. Acquisition of the Crosstalk Targets of the Disease and Medicine. The corresponding targets of Chansu injection related to COVID-19 were mapped using the $\mathrm{R}$ language data package (version 3.6.1). The crosstalk targets were considered potential targets for COVID-19 treatment with Chansu injection.
2.6. Network Construction. A medicine-target-disease network diagram was prepared by introducing the acquired information of crosstalk targets and active ingredients into the Cytoscape software (v3.7.2). Thereafter, a topological analysis was performed.

2.7. Acquisition of the PPI Diagram. The acquired crosstalk targets were recorded in the STRING database using the genus Homo sapiens. The free targets were deleted, and the resultant file, string_interactions.tsv, was introduced into the Cytoscape software for topological analysis. Core targets were determined according to the degree and centrality of the intermediate value.

2.8. GO and KEGG Enrichment Analysis. The function of the core targets was described and annotated to investigate its pathway. Kyoto Encyclopedia of Genes and Genomes (KEGG) and Gene Ontology (GO) enrichment analyses were conducted using the DAVID database. For GO enrichment analysis, molecular function (MF), biological process (BP), 
and cellular component (CC) were used to confine and describe genes. The potential signaling pathway for COVID19 treatment using Chansu injection was determined through KEGG enrichment analysis.

2.9. Molecular Docking. The molecular structure of the active ingredients of Chansu injection was downloaded from PubChem, while those of 3CL protease, ACE2, RdRp, and spike protein were downloaded from the PDB database. The data were processed using MGLTools 1.5.6 and saved as a pdbqt file. The active sites and grid box coordinates of 3CL protease, ACE2, RdRp, and spike protein were defined according to their built-in ligands. The size of each box was $30 \times 30 \times 30$ grids. Small-molecule proteins were docked using AutoDock Vina 1.1.2; molecular structures were stereotyped using PyMOL; and the top scoring conformations were mapped and analyzed using Maestro 11.9.

We used ChemBioDraw Ultra 17.0 to draw the structures of compounds, which were then converted to $3 \mathrm{D}$ structures using ChemBio3D Ultra 17.0 and optimized using MMFF94 force fields. The 3D structures of RdRp, 3CL, ACE2, and spike were downloaded from the RCSB Protein Data Bank (https://www.rcsb.org), and we selected the 3D structures of RdRp (PDB ID: 6ld3), 3CL (PDB ID: 6LU7), ACE2 (PDB ID: 1R42), and spike (PDB ID: 6acc) were used as the proteins for docking in this project. Both proteins and compounds were converted to PDBQT format using AutoDockTools 1.5.6 [29, 30]. AutoDock Vina 1.1.2 [31] was used for the molecular docking study. To increase the accuracy of the calculations, we set the parameter exhaustiveness to 20. The default values were used for all parameters except where noted. Finally, the conformation with the highest scoring value was selected for analysis of the results using Free Maestro 11.9.

\section{Results}

3.1. Active Ingredients of Chansu Injection. The major components of Chansu injection were analyzed using HPLC. On comparison with standard reference compounds, three compounds were identified: bufalin, resibufogenin, and cinobufagin. Representative chromatograms of the standard reference compounds (Figure 2(a)) and Chansu injection components (Figure 2(b)) are shown in Figure 2.

3.2. Potential Targets of Chansu Injection. The active ingredients of bufalin, resibufogenin, and cinobufagin were searched online in PharmMapper, SEA, and Swiss databases, and the results were combined and duplicates were eliminated to obtain a total of 236 potential targets.

\subsection{Crosstalk Targets of Chansu Injection and COVID-19.} By searching GeneCards, CTD, and TTD, a total of 16,611 disease-related targets were obtained (7,927 from GeneCards, 14,774 from CTD, and 2 from TTD). When the potential targets of Chansu injection were mapped to disease-related targets, a total of 77 crosstalk targets were identified. In particular, COVID-19 was found to have 96, 99, and 92 crosstalk targets with bufalin, cinobufagin, and resibufogenin, respectively (Figure 3 ).

3.4. Medicine-Target-Disease Network Diagram. In the medicine-target-disease network diagram (Figure 4), the outer light blue nodes represent targets, the inner dark blue nodes represent the active ingredients of Chansu injection, the red node represents medicines, and the green node represents diseases. Topological analysis of the network diagram showed that there were 82 nodes and 137 connecting lines. Furthermore, the network centralization, heterogeneity, and density were 0.199, 1.096, and 0.032, respectively. This suggests an important link between Chansu injection and COVID-19 and motivated us to proceed to the next step in our analysis.

3.5. PPI Diagram and Core Target Filtration. Information concerning the crosstalk targets of the disease and drug was introduced into the STRING database for analysis using the genus Homo sapiens. Four free targets were deleted, and the resultant file "string_interactions.tsv" was introduced into the Cytoscape software (v3.7.2) to draw the protein-protein interaction (PPI) diagram. Thereafter, a topological analysis was performed. The nodes with retention value and the centrality of intermediate values above the mean values are the core targets (Figure 5).

As shown in Table 1, core targets were obtained, namely, AKT2, GRB2, EGFR, CCNA2, RXRB, RARA, STAT1, PPARG, ESR1, SRC, NOS3, IL2, PPARA, MMP2, MDM2, and CASP3.

3.6. GO and KEGG Enrichment Analysis. Based on the GO enrichment analysis, a total of 26 entries had a $P$ value less than 0.05 , including $16 \mathrm{BP}, 4 \mathrm{CC}$, and $6 \mathrm{MF}$ (Figure 6). These entries are involved in cell proliferation, modulation of apoptosis and programmed death, cascading response of intracellular signals, immune responses, nucleic acid metabolism processes, transcription, and translation. Based on the KEGG enrichment analysis, the phosphoinositide 3kinase (PI3K-Akt) and JAK-STAT signaling pathways differed significantly and were closely related to viral infection (Figure 7).

3.7. Molecular Docking. The 3CL sequence is highly conserved in coronaviruses and is essential for the normal function of SARS-CoV-2 [32]. The high affinity of ACE2 for the extracellular domain structure of the novel coronavirus $S$ protein is $15 \mathrm{nM}$ (equilibrium dissociation constant), which is 10-20 times higher than the affinity of ACE2 for the extracellular domain structure of the SARS coronavirus $S$ protein [33]. The high affinity of ACE2 may help the virus to spread easily from human to human, and it is therefore an important target.

As shown in Figure 8, bufalin had the highest binding efficiency to 3CL protease, ACE2, RNA-dependent RNA polymerase (RdRp), and spike protein, with an absolute 


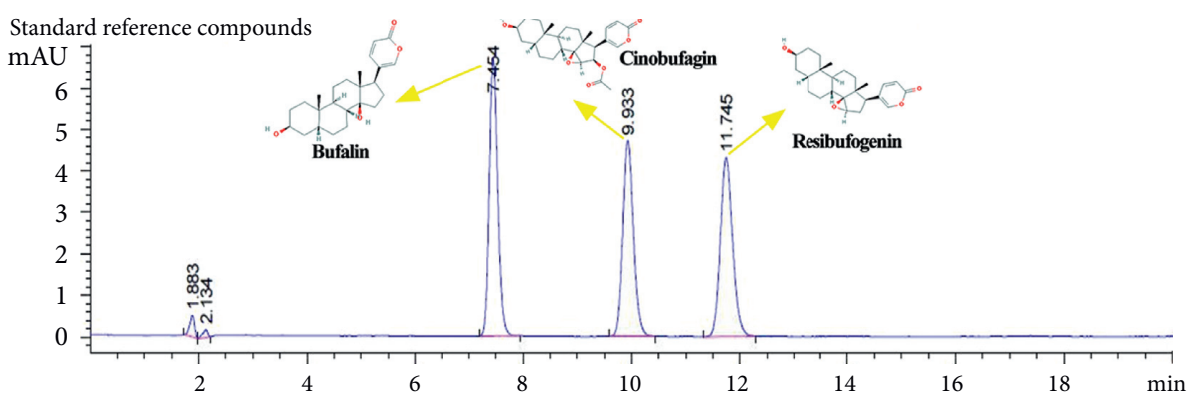

(a)

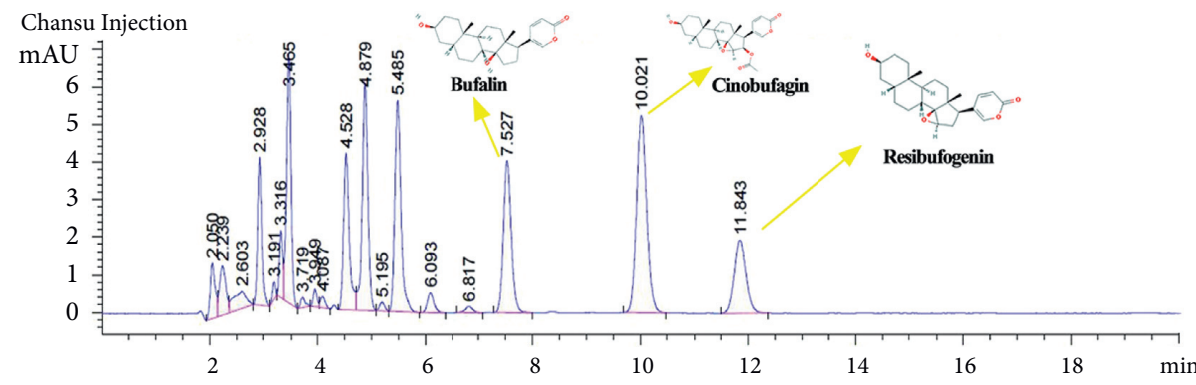

(b)

FIgURE 2: HPLC chromatograms of the components of Chansu injection.

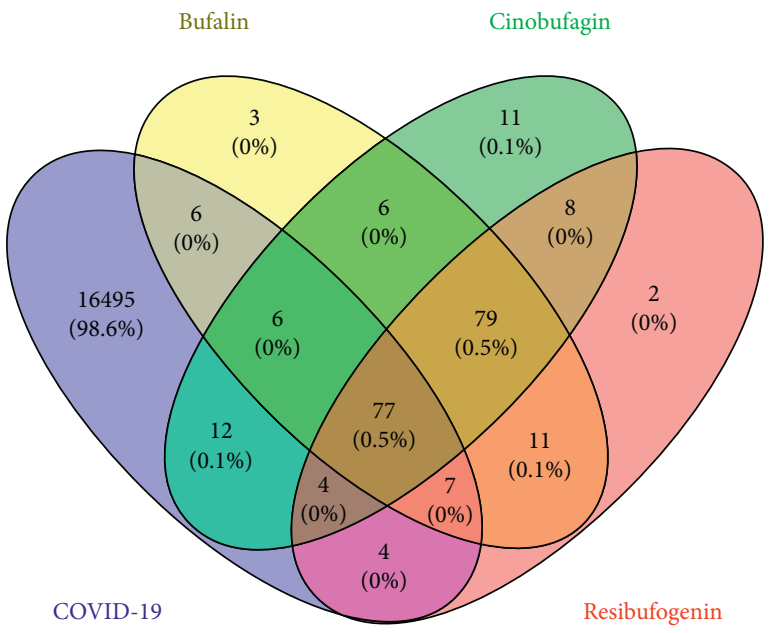

FIgURe 3: Venn diagram.

binding efficiency above 7, indirectly suggesting that the Chansu injection has a good therapeutic effect on SARSCoV-2. The molecular docking diagram of bufalin and virus proteins is shown in Figure 9.

\section{Discussion}

COVID-19 is a global pandemic in the $21^{\text {st }}$ century that has greatly threatened human health and safety [34]. Chinese medicines can enhance the self-immunity and reconstruction capacity of the human body when used to prevent infectious diseases, thereby maintaining homeostasis [35]. They function to balance immunity, eliminate inflammation, and avoid or delay inflammation outburst by regulating immunity, inflammation, endocrine function, signal

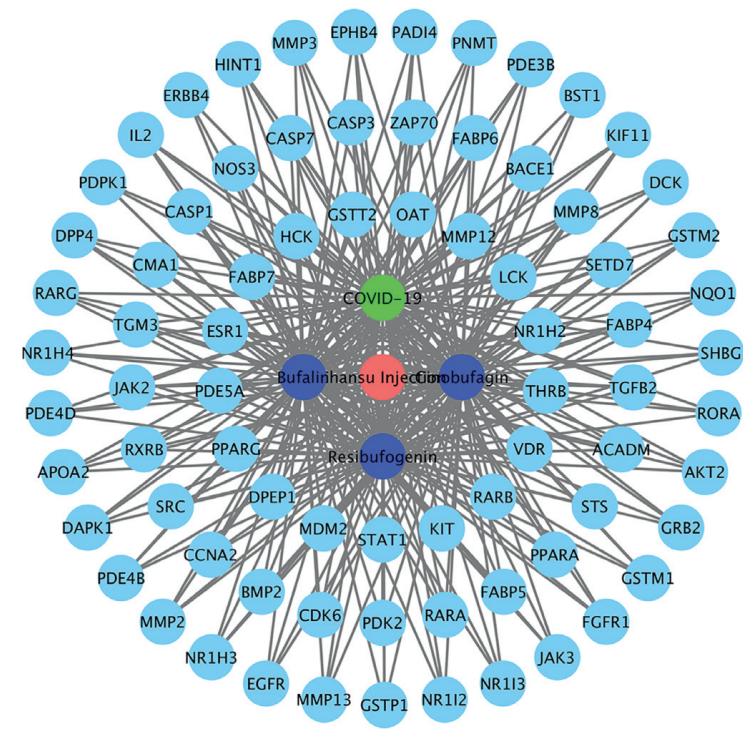

FIgURE 4: Network diagram of the drug-target-disease.

transduction, and other BPs and inhibiting the production of bacterial endotoxins through multiple ingredients and targets [36]. Chinese medicines have a unique advantage and provide effective control strategies in severe conditions, such as global pandemics, which lack effective treatments.

Previously, bufalin, the main active ingredient of Chansu, was found to have a broad-spectrum antiviral activity that can inhibit mouse hepatitis virus, feline infectious peritonitis virus, MERS-CoV, and vesicular stomatitis virus infecting host cells via the ATP1A1-mediated Src signaling pathway $[37,38]$. In addition, ATP1A1 plays a crucial role in the infection of host cells by Ebola and respiratory syncytial virus (RSV) [39, 40]. 


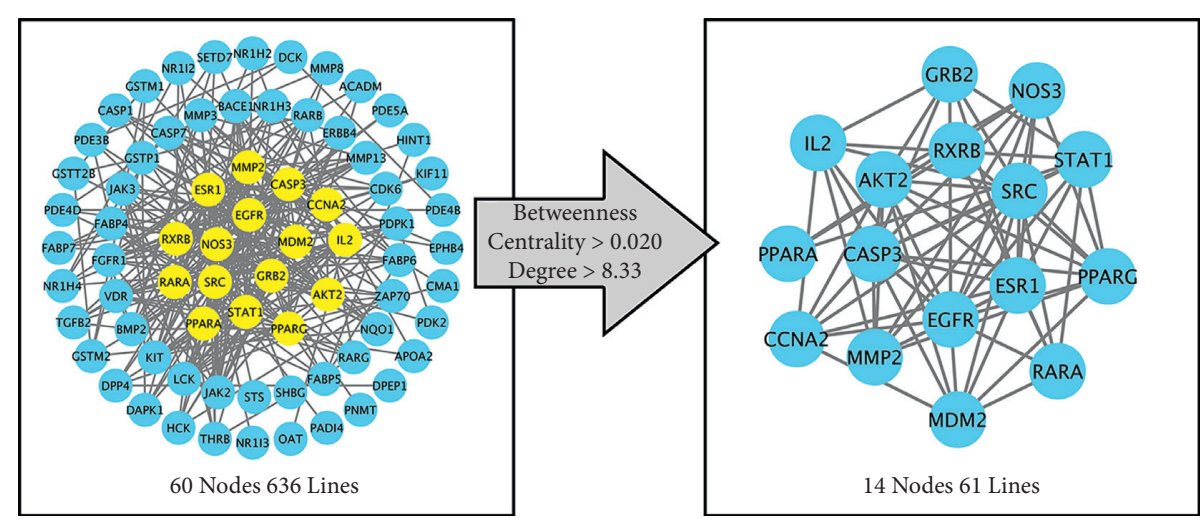

Figure 5: PPI diagram and filtration of the core targets.

TABLE 1: Core targets of Chansu injection.

\begin{tabular}{lcc}
\hline Gene name & Protein name & UniProt ID \\
AKT2 & RAC-beta serine/threonine-protein kinase & P31751 \\
GRB2 & Growth factor receptor-bound protein 2 & P62993 \\
EGFR & Epidermal growth factor receptor & P00533 \\
CCNA2 & Cyclin-A2 & P20248 \\
RXRB & Retinoic acid receptor RXR-beta & P28702 \\
RARA & Retinoic acid receptor alpha & P10276 \\
STAT1 & Signal transducer and activator of transcription 1-alpha/beta & P42224 \\
PPARG & Peroxisome proliferator-activated receptor gamma & P37231 \\
ESR1 & Estrogen receptor & P03372 \\
SRC & Proto-oncogene tyrosine-protein kinase Src & P12931 \\
NOS3 & Nitric oxide synthase \\
IL2 & Interleukin-2 & P29474 \\
PPARA & Peroxisome proliferator-activated receptor alpha & P60568 \\
MMP2 & 72 kDa type IV collagenase & Q07869 \\
MDM2 & E3 ubiquitin-protein ligase Mdm2 & Q08253 \\
CASP3 & Caspase-3 & P42574 \\
\hline
\end{tabular}

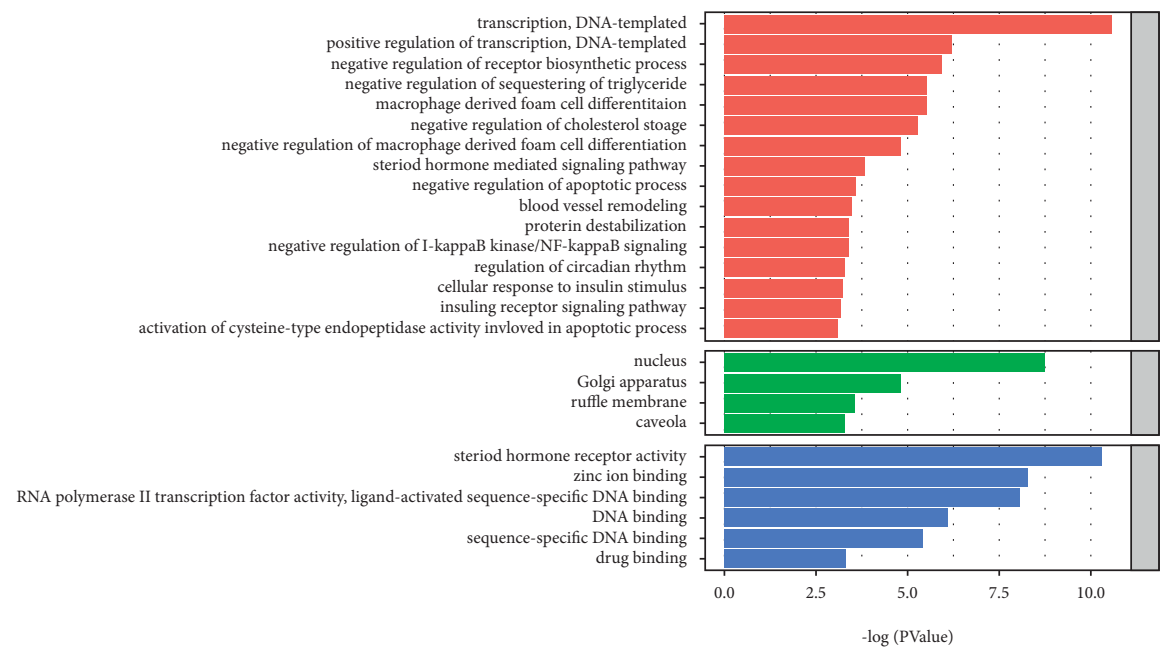

Figure 6: Results of GO enrichment analysis.

A 50-sample clinical [24] study showed that Chansu injection could significantly improve the respiratory function of patients with severe COVID-19. In fact, the $\mathrm{PaO}_{2} /$ $\mathrm{FiO}_{2}$ and $\mathrm{ROX}$ of $95.2 \%$ patients in the treatment group were improved, while those in the control group improved by
$68.4 \%$ and $73.7 \%$, respectively. The respiration-supported recovery time of the treatment group was one day shorter than that of the control group. Meanwhile, the results of the safety index analysis showed that Chansu injection did not have evident toxic side effects at the tested dosage. 


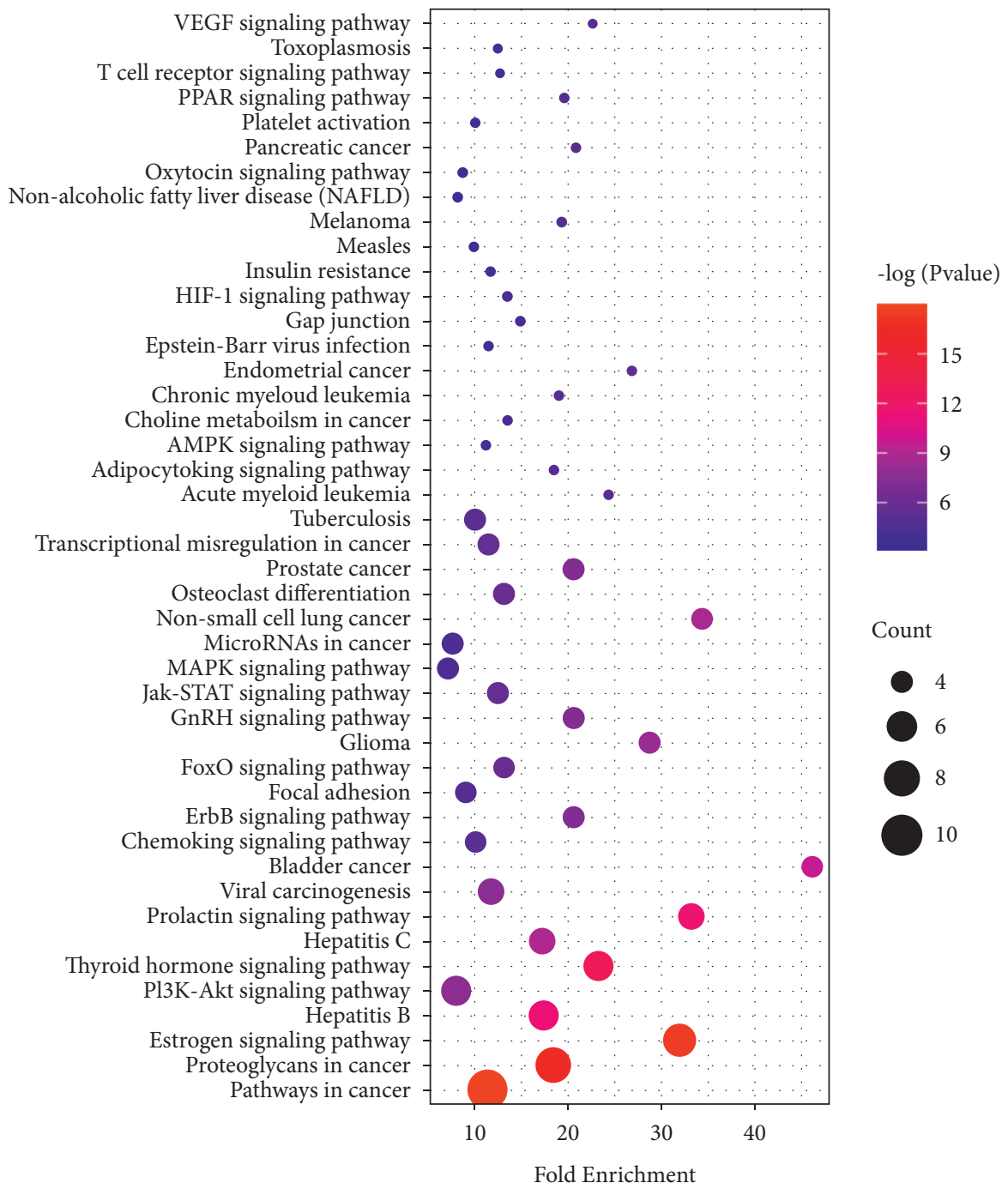

FIGURE 7: Results of KEGG enrichment analysis.

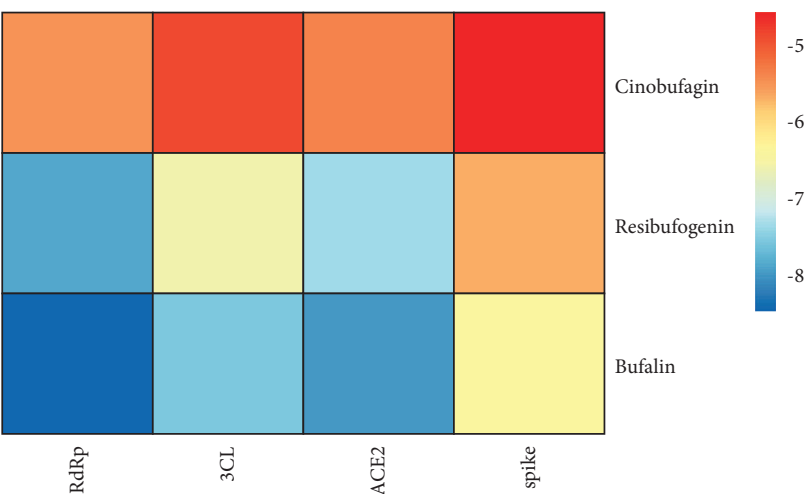

FIGURE 8: Diagram of the molecular docking efficiency.

As Chansu injection can effectively treat COVID-19, network pharmacology was used to study the molecular mechanism of Chansu injection, as with other comprehensive pharmacological analyses of medicinal plants [25], which might be involved in the regulation of cell proliferation, cell apoptosis, programmed cell death, intracellular signaling cascade response, immune response, nucleic acid metabolism, transcription, translation, and other BPs in COVID-19 treatment. Based on the KEGG enrichment analysis results, the PI3K-Akt and JAK-STAT signaling pathways may play a critical role. Furthermore, the molecular docking results suggested that bufalin, one of the active ingredients in Chansu injection, has a very high binding efficiency to 3CL protease, ACE2, and RdRp, and spike protein, with an absolute binding efficiency above 7, suggesting that the active ingredients in Chansu injection may have stopped viral entry into host cells and blocked its binding to ACE2 and the binding of the SARS-CoV-2 protein to the hydrolase of SARS-CoV-2 3CL protease, thereby terminating viral RNA duplication. This study has laid the foundation for future confirmation of the molecular mechanisms of Chansu injection in COVID-19 treatment.

At present, the role of the PI3K-Akt signaling pathway in various diseases has been confirmed. In humans, the PI3KAkt pathway regulates multiple cellular and biological processes, such as tumor immunity, virus infection, and inflammatory recovery. PI3K is an intracellular 

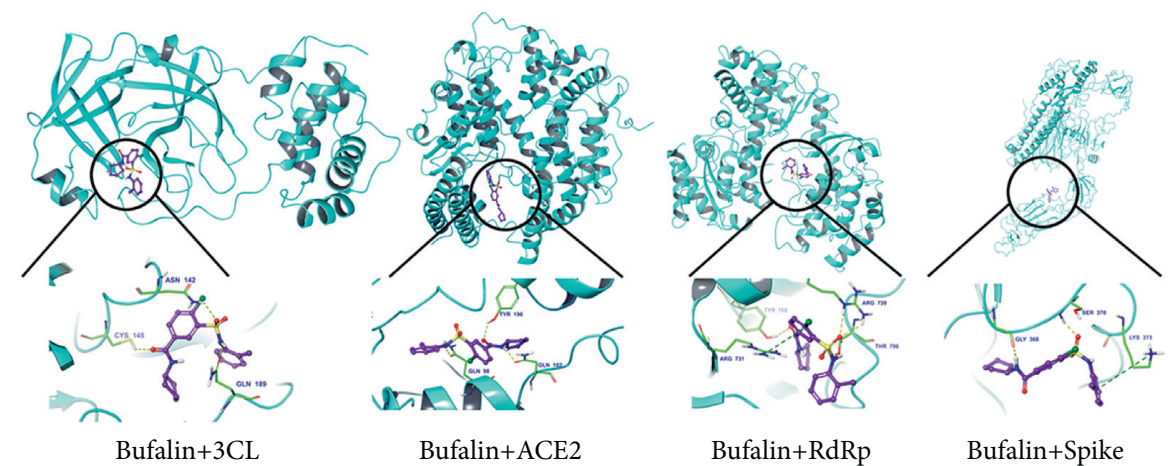

FIGURE 9: Molecular docking of Chansu injection to 3CL protease, ACE2, RdRp, and spike protein.

phosphatidylinositol kinase (PIK) that also has Ser/Thr kinase and PIK activities, which participate in the regulation of various cellular functions, such as proliferation, differentiation, and glucose transportation [41, 42]. The PI3K-Akt signaling pathway was found to be involved in the infection processes of different types of viruses, including RNA and DNA viruses, which mainly regulate viral activity in the early infection stage, recognize host cell infection, and mediate cell transformation [43-46].

Iranian scholars found that viruses depend on two types of receptors to enter cells: ACE2 and CD147 [47]. Other molecular factors on the cell surface that facilitate viral genome entry into host cells include TMPRSS2 and furin protease. CD147 and furin protease both help induce the PI3K/Akt signal transduction pathway. Furthermore, the endocytosis of SARS-CoV-2 is mediated by clathrin, and this pathway is regulated by $\mathrm{PI} 3 \mathrm{~K} / \mathrm{Akt}$ signal transduction. Once the virus binds to ACE2, the cell membrane sinks and viral endocytosis occurs. A reduction of ACE2 on the cell surface helps increase the level of serum angiotensin II (Ang II), which has been proven in COVID-19 patients. However, the binding of Ang II to its type I receptor (AT1R) activates this signaling pathway, thereby regulating the SARS-CoV-2 infection to host cells and virus duplication in infected cells. Therefore, the PI3K/Akt signaling pathway could regulate the activation of activator protein$1(\mathrm{AP}-1)$ and nucleic factor $-\kappa \mathrm{B}(\mathrm{NF}-\kappa \mathrm{B})$, reduce the expression of inflammatory cytokines such as interleukin- 6 (IL-6) and tumor necrosis factor alpha (TNF- $\alpha$ ), inhibit the cascading reaction of an inflammatory outburst, and promote the absorption of inflammatory cells and tissue recovery.

The JAK/STAT signaling pathway plays a crucial role in the regulation of cell growth, survival, and pathogen resistance [48-51]. Professor Yaoxing Wu et al. [52] found that type I interferon pretreatment could significantly interfere with the duplication of SARS-CoV-2, suggesting a certain sensitivity of SARS-CoV-2 to type I interferon cells. The induced interferon was released to regulate the JAK-STAT-mediated expression of multiple downstream antiviral cytokines (such as IFN- $\gamma$ and IL-3) and inhibit virus duplication and amplification. The $\mathrm{M}^{\text {pro }}$ in SARS-CoV-2 could promote autophagic degradation of the transcriptomic factor STAT1 to weaken the entrance of STAT1 into the nucleus. This process inhibits the activity of type I interferon cells, reduces the expression of the downstream active ingredient of the JAK-STAT pathway, and helps COVID-19 patients acquire a natural immune escape.

A study [53] by June's research group at the University of Pennsylvania (USA) found that the cytokine release syndrome (CRS) is the primary lethal cause for SARS and MERS patients, as well as COVID-19 patients. When a coronavirus enters the body, monocytes, macrophages, and dendritic cells secrete IL- 6 and other inflammatory factors. The downstream activity of IL-16 mainly includes the classical cis and trans pathways. In the cis pathway, IL-6 forms a complex with the MIL- 6 membrane and gp130 and activates the JAK and STAT3 pathways. MIL-6 is mainly expressed in immune cells; therefore, this pathway can activate immune cells to trigger CRS. In the trans pathway, the highly concentrated IL-6 binds to the soluble IL-6R and forms a dipolymer with gp130. As gp130 is widely expressed on the surface of all cells, the downstream JAKSTAT3 pathway can activate cells that do not express MIL$6 \mathrm{R}$, such as epithelial cells. Furthermore, they may increase levels of vascular endothelial growth factor (VEGF), monocyte chemotactic protein-1 (MCP-1), and interleukin-8 (IL-8) levels, as well as release of IL-6 and reduction of E-cadherin, eventually leading to acute respiratory distress syndrome (ARDS).

\section{Conclusion}

In summary, in this study, we determined the binding efficiency of Chansu injection to the main protein of the novel coronavirus and explored the functioning mechanism of this Chinese medicine for COVID-19 treatment using network pharmacology and molecular docking based on highly effective clinical studies. The results showed that Chansu injection could regulate virus duplication, activate immune cells, and alleviate inflammatory responses through the PI3K-Akt and JAK-STAT signaling pathways. The Chansu injection treats patients with COVID-19 by acting on SARS- 
CoV-2 and ACE2. The results of this study provide theoretical and experimental evidence for COVID-19 treatment using Chansu injection.

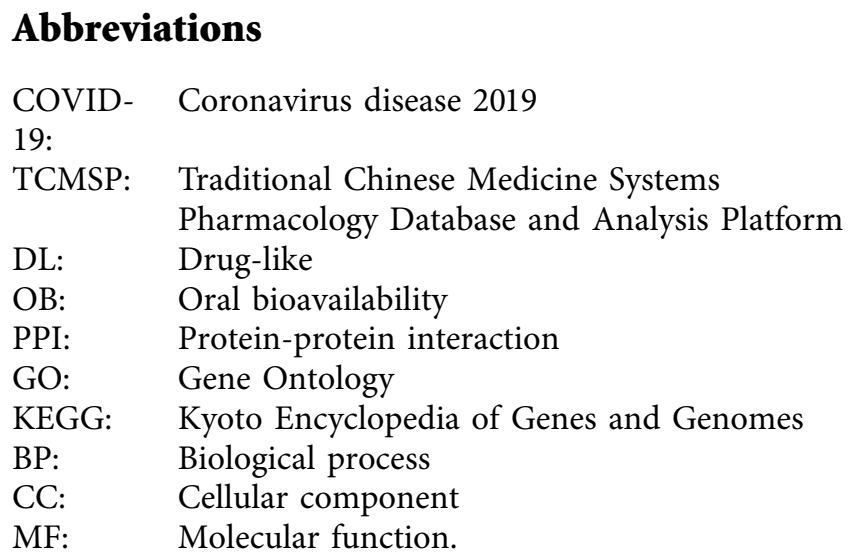

\section{Data Availability}

The data and materials used to support the findings of this study are available from the corresponding author upon request.

\section{Ethical Approval}

Not applicable.

\section{Consent}

Not applicable.

\section{Disclosure}

An earlier version of it has been presented as "Pre-print" in research square according to the following link: https://www.researchsquare.com/article/rs-364843/v1.

\section{Conflicts of Interest}

The authors declare that they have no conflicts of interest.

\section{Authors' Contributions}

The authors Yong Xu and Wenpan Peng contributed equally to this work. XZ and QW designed the study. YX, WP, DH, $\mathrm{WZ}$, and FF performed the experiments. YX and WP analyzed the data. YX, WP, and DH wrote the main manuscript. XZ and QW were responsible for quality control of the study. All authors reviewed the manuscript.

\section{Acknowledgments}

The authors thank Editage (https://www.editage.cn) for English language editing. This work was supported by the National Natural Science Foundation of China (82074358) and Jiangsu Province's Colleges and Universities (integration of Chinese and Western medicine).

\section{Supplementary Materials}

Supplementary Table 1. Detailed data of association and attribution of genes, ingredients, medicine, and diseases in Figure 4. (Supplementary Materials)

\section{References}

[1] D. Lenihan, J. Carver, C. Porter et al., "Cardio-oncology care in the era of the coronavirus disease 2019 (COVID-19) pandemic: an International Cardio-Oncology Society (ICOS) statement," CA: A Cancer Journal for Clinicians, vol. 70, no. 6, pp. $480-504,2020$.

[2] W. J. Wiersinga, A. Rhodes, A. C. Cheng, S. J. Peacock, and H. C. Prescott, "Pathophysiology, transmission, diagnosis, and treatment of coronavirus disease 2019 (COVID-19)," Journal of the American Medical Association, vol. 324, no. 8, pp. 782-793, 2020.

[3] A. Esmaeilzadeh and R. Elahi, "Immunobiology and immunotherapy of COVID-19: a clinically updated overview," Journal of Cellular Physiology, vol. 236, no. 4, pp. 2519-2543, 2021.

[4] N. Le Bert, A. T. Tan, K. Kunasegaran et al., "SARS-CoV-2specific T cell immunity in cases of COVID-19 and SARS, and uninfected controls," Nature, vol. 584, no. 7821, pp. 457-462, 2020.

[5] P. K. Samudrala, P. Kumar, K. Choudhary et al., "Virology, pathogenesis, diagnosis and in-line treatment of COVID-19," European Journal of Pharmacology, vol. 883, Article ID 173375, 2020.

[6] M. Yüce, E. Filiztekin, and K. G. Özkaya, "COVID-19 diagnosis-a review of current methods," Biosensors and Bioelectronics, vol. 172, Article ID 112752, 2021.

[7] A. Wilder-Smith and S. Osman, "Public health emergencies of international concern: a historic overview," Journal of Travel Medicine, vol. 27, no. 8, 2020.

[8] J. Majumder and T. Minko, "Recent developments on therapeutic and diagnostic approaches for COVID-19," The AAPS Journal, vol. 23, no. 1, p. 14, 2021.

[9] N. Taleghani and F. Taghipour, "Diagnosis of COVID-19 for controlling the pandemic: a review of the state-of-the-art," Biosensors and Bioelectronics, vol. 174, Article ID 112830, 2021.

[10] N. Petrosillo, G. Viceconte, O. Ergonul, G. Ippolito, and E. Petersen, "COVID-19, SARS and MERS: are they closely related?" Clinical Microbiology and Infections, vol. 26, no. 6, pp. 729-734, 2020.

[11] K. Dhama, S. Khan, R. Tiwari et al., "Coronavirus disease 2019-COVID-19," Clinical Microbiology Reviews, vol. 33, no. $4,2020$.

[12] Y.-Y. Zhang, B.-R. Li, and B.-T. Ning, "The comparative immunological characteristics of SARS-CoV, MERS-CoV, and SARS-CoV-2 coronavirus infections," Frontiers in Immunology, vol. 11, p. 2033, 2020.

[13] C. Ronco, T. Reis, and F. Husain-Syed, "Management of acute kidney injury in patients with COVID-19," The Lancet Respiratory Medicine, vol. 8, no. 7, pp. 738-742, 2020.

[14] L. Piroth, J. Cottenet, A.-S. Mariet et al., "Comparison of the characteristics, morbidity, and mortality of COVID-19 and seasonal influenza: a nationwide, population-based retrospective cohort study," The Lancet Respiratory Medicine, vol. 9, no. 3, pp. 251-259, 2021.

[15] A. Hazafa, K. Ur-Rahman, I.-U. Haq et al., "The broadspectrum antiviral recommendations for drug discovery 
against COVID-19," Drug Metabolism Reviews, vol. 52, no. 3 , pp. 408-424, 2020.

[16] M. Bayat, Y. Asemani, and S. Najafi, "Essential considerations during vaccine design against COVID-19 and review of pioneering vaccine candidate platforms," International Immunopharmacology, vol. 97, Article ID 107679, 2021.

[17] M. Jeyanathan, S. Afkhami, F. Smaill, M. S. Miller, B. D. Lichty, and Z. Xing, "Immunological considerations for COVID-19 vaccine strategies," Nature Reviews Immunology, vol. 20, no. 10, pp. 615-632, 2020.

[18] Y.-F. Huang, C. Bai, F. He, Y. Xie, and H. Zhou, "Review on the potential action mechanisms of Chinese medicines in treating Coronavirus disease 2019 (COVID-19)," Pharmacological Research, vol. 158, Article ID 104939, 2020.

[19] K. Hu, W.-J. Guan, Y. Bi et al., "Efficacy and safety of Lianhuaqingwen capsules, a repurposed Chinese herb, in patients with coronavirus disease 2019: a multicenter, prospective, randomized controlled trial," Phytomedicine, vol. 85, Article ID 153242, 2020.

[20] J. Wang, H. Cai, Q. Liu et al., "Cinobufacini inhibits colon cancer invasion and metastasis via suppressing wnt $/ \beta$-catenin signaling pathway and EMT," The American Journal of Chinese Medicine, vol. 48, no. 3, pp. 703-718, 2020.

[21] L.-J. Deng, Y.-H. Lei, J.-Y. Quan et al., " $1 \beta-\mathrm{OH}$-arenobufagin induces mitochondrial apoptosis in hepatocellular carcinoma through the suppression of mTOR signaling pathway," Journal of Ethnopharmacology, vol. 266, Article ID 113443, 2021.

[22] J. Qi, A. H. M. Zulfiker, C. Li, D. Good, and M. Q. Wei, “The development of toad toxins as potential therapeutic agents," Toxins, vol. 10, no. 8, 2018.

[23] X. Zhan, H. Wu, H. Wu et al., "Metabolites from Bufo gargarizans (Cantor, 1842): a review of traditional uses, pharmacological activity, toxicity and quality control," Journal of Ethnopharmacology, vol. 246, Article ID 112178, 2020.

[24] F. Hu, J. Chen, H. Chen et al., "Chansu injection improves the respiratory function of severe COVID-19 patients," MedRxiv, 2020.

[25] K. K. Oh, M. Adnan, and D. H. Cho, "Network pharmacology approach to decipher signaling pathways associated with target proteins of NSAIDs against COVID-19," Scientific Reports, vol. 11, no. 1, p. 9606, 2021.

[26] K. K. Oh, M. Adnan, and D. H. Cho, "Active ingredients and mechanisms of Phellinus linteus (grown on Rosa multiflora) for alleviation of Type 2 diabetes mellitus through network pharmacology," Gene, vol. 768, Article ID 145320, 2021.

[27] T.-T. Luo, Y. Lu, S.-K. Yan, X. Xiao, X.-l. Rong, and J. Guo, "Network pharmacology in research of Chinese medicine formula: methodology, application and prospective," Chinese Journal of Integrative Medicine, vol. 26, no. 1, pp. 72-80, 2020.

[28] L. Pinzi and G. Rastelli, "Molecular docking: shifting paradigms in drug discovery," International Journal of Molecular Sciences, vol. 20, no. 18, 2019.

[29] M. F. Sanner, "Python: a programming language for software integration and development," Journal of Molecular Graphics \& Modelling, vol. 17, no. 1, pp. 57-61, 1999.

[30] G. M. Morris, R. Huey, W. Lindstrom et al., "AutoDock4 and AutoDockTools4: automated docking with selective receptor flexibility," Journal of Computational Chemistry, vol. 30, no. 16, pp. 2785-2791, 2009.

[31] O. Trott and A. J. Olson, "AutoDock Vina: improving the speed and accuracy of docking with a new scoring function, efficient optimization, and multithreading," Journal of Computational Chemistry, vol. 31, no. 2, pp. 455-461, 2010.
[32] D. Wrapp, N. Wang, K. S. Corbett et al., "Cryo-EM structure of the 2019-nCoV spike in the prefusion conformation," BioRxiv, vol. 13, no. 6483, pp. 1260-1263, 2020.

[33] D. Wrapp, N. Wang, K. S. Corbett et al., "Cryo-EM structure of the 2019-nCoV spike in the prefusion conformation," Science, vol. 367, pp. 1260-1263, 2020.

[34] C. L. Atzrodt, I. Maknojia, R. D. P. McCarthy et al., "A Guide to COVID-19: a global pandemic caused by the novel coronavirus SARS-CoV-2," FEBS Journal, vol. 287, no. 17, pp. 3633-3650, 2020.

[35] K. W. Chan, V. T. Wong, and S. C. W. Tang, "COVID-19: an update on the epidemiological, clinical, preventive and therapeutic evidence and guidelines of integrative Chinesewestern medicine for the management of 2019 novel coronavirus disease," The American Journal of Chinese Medicine, vol. 48, no. 3, pp. 737-762, 2020.

[36] F. Huang, Y. Li, E. L.-H. Leung et al., "A review of therapeutic agents and Chinese herbal medicines against SARS-COV-2 (COVID-19)," Pharmacological Research, vol. 158, Article ID 104929, 2020.

[37] F. Qi, Z. X. Wang, P. P. Cai et al., "Traditional Chinese medicine and related active compounds: a review of their role on hepatitis B virus infection," Drug Discoveries \& Therapeutics, vol. 7, no. 6, pp. 212-224, 2013.

[38] Z.-R. Zhang, Y.-N. Zhang, X.-D. Li et al., "A cell-based largescale screening of natural compounds for inhibitors of SARSCoV-2," Signal Transduction and Targeted Therapy, vol. 5, no. 1, p. 218, 2020.

[39] I. García-Dorival, W. Wu, S. Dowall et al., "Elucidation of the Ebola virus VP24 cellular interactome and disruption of virus biology through targeted inhibition of host-cell protein function," Journal of Proteome Research, vol. 13, no. 11, pp. 5120-5135, 2014.

[40] M. Lingemann, T. McCarty, X. Liu et al., "The alpha-1 subunit of the $\mathrm{Na}+\mathrm{K}+$-ATPase (ATP1A1) is required for macropinocytic entry of respiratory syncytial virus (RSV) in human respiratory epithelial cells," PLoS Pathogens, vol. 15, no. 8, Article ID e1007963, 2019.

[41] M. Aoki and T. Fujishita, "Oncogenic roles of the PI3K/AKT/ mTOR axis," Current Topics in Microbiology and Immunology, vol. 407, pp. 153-189, 2017.

[42] Q. Yang, W. Jiang, and P. Hou, "Emerging role of PI3K/AKT in tumor-related epigenetic regulation," Seminars in Cancer Biology, vol. 59, pp. 112-124, 2019.

[43] T. Okamoto, T. Suzuki, S. Kusakabe et al., "Regulation of apoptosis during flavivirus infection," Viruses, vol. 9, no. 9, 2017.

[44] H. Li, J. Zhu, M. He, Q. Luo, F. Liu, and R. Chen, "Marek's disease virus activates the PI3K/akt pathway through interaction of its protein meq with the P85 subunit of PI3K to promote viral replication," Frontiers in Microbiology, vol. 9, p. 2547, 2018.

[45] F. Bossler, K. Hoppe-Seyler, and F. Hoppe-Seyler, "PI3K/ $\mathrm{AKT} / \mathrm{mTOR}$ signaling regulates the virus/host cell crosstalk in HPV-positive cervical cancer cells," International Journal of Molecular Sciences, vol. 20, no. 9, 2019.

[46] Y. Zhan, S. Yu, S. Yang et al., "Newcastle disease virus infection activates PI3K/Akt/mTOR and p38 MAPK/Mnk1 pathways to benefit viral mRNA translation via interaction of the viral NP protein and host eIF4E," PLoS Pathogens, vol. 16, no. 6, Article ID e1008610, 2020.

[47] M. R. Khezri, "PI3K/AKT signaling pathway: a possible target for adjuvant therapy in COVID-19," Human Cell, vol. 34, no. 2, pp. 700-701, 2021. 
[48] A. V. Villarino, Y. Kanno, and J. J. O'Shea, "Mechanisms and consequences of Jak-STAT signaling in the immune system," Nature Immunology, vol. 18, no. 4, pp. 374-384, 2017.

[49] R. Morris, N. J. Kershaw, and J. J. Babon, "The molecular details of cytokine signaling via the JAK/STAT pathway," Protein Science, vol. 27, no. 12, pp. 1984-2009, 2018.

[50] S. Kaur, Y. Bansal, R. Kumar, and G. Bansal, "A panoramic review of IL-6: structure, pathophysiological roles and inhibitors," Bioorganic \& Medicinal Chemistry, vol. 28, no. 5, Article ID 115327, 2020.

[51] P. Xin, X. Xu, C. Deng et al., "The role of JAK/STAT signaling pathway and its inhibitors in diseases," International Immunopharmacology, vol. 80, Article ID 106210, 2020.

[52] Y. Wu, L. Ma, Z. Zhuang et al., "Main protease of SARS-CoV2 serves as a bifunctional molecule in restricting type I interferon antiviral signaling," Signal Transduction and Targeted Therapy, vol. 5, no. 1, p. 221, 2020.

[53] J. B. Moore and C. H. June, "Cytokine release syndrome in severe COVID-19," Science, vol. 368, no. 6490, pp. 473-474, 2020 . 